\title{
Vocal Music Listening Enhances Poststroke Language Network Reorganization
}

\author{
(1)Aleksi J. Sihvonen, ${ }^{1,2}$ Pablo Ripollés, ${ }^{3,4,5}$ Vera Leo, ${ }^{1}$ Jani Saunavaara, ${ }^{6}$ Riitta Parkkola, \\ Antoni Rodríguez-Fornells, ${ }^{8,9,10}$ Seppo Soinila, ${ }^{11}$ and ${ }^{1}$ Teppo Särkämö ${ }^{1}$
}

\section{https://doi.org/10.1523/ENEURO.0158-21.2021}

\begin{abstract}
${ }^{1}$ Cognitive Brain Research Unit, Department of Psychology and Logopedics, Faculty of Medicine, University of Helsinki, FI-00014 Helsinki, Finland, ${ }^{2}$ Centre for Clinical Research, The University of Queensland, Herston 4029, Queensland, Australia, ${ }^{3}$ Department of Psychology, New York University, New York, New York 10003, ${ }^{4}$ Music and Audio Research Laboratory, New York University, Brooklyn, New York 11201, ${ }^{5}$ Center for Language, Music and Emotion, New York University, New York, New York 10014, ${ }^{6}$ Department of Medical Physics, Turku University Hospital, 20521 Turku, Finland, ${ }^{7}$ Department of Radiology, Turku University Hospital and University of Turku, FI-20520 Turku, Finland, ${ }^{8}$ Cognition and Brain Plasticity Group, Bellvitge Biomedical Research Institute, L'Hospitalet de Llobregat, 08908 Barcelona, Spain, ${ }^{9}$ Department of Cognition, Development and Education Psychology, University of Barcelona, 08035 Barcelona, Spain, ${ }^{10}$ Institució Catalana de Recerca i Estudis Avançats, 08037 Barcelona, Spain, and ${ }^{11}$ Neurocenter, Turku University Hospital and Division of Clinical Neurosciences, University of Turku, 20521 Turku, Finland
\end{abstract}

\begin{abstract}
Listening to vocal music has been recently shown to improve language recovery in stroke survivors. The neuroplasticity mechanisms supporting this effect are, however, still unknown. Using data from a three-arm, single-blind, randomized controlled trial including acute stroke patients $(N=38)$ and a 3 month follow-up, we set out to compare the neuroplasticity effects of daily listening to self-selected vocal music, instrumental music, and audiobooks on both brain activity and structural connectivity of the language network. Using deterministic tractography, we show that the 3 month intervention induced an enhancement of the microstructural properties of the left frontal aslant tract (FAT) for the vocal music group compared with the audiobook group. Importantly, this increase in the strength of the structural connectivity of the left FAT correlated with improved language skills. Analyses of stimulus-specific activation changes showed that the vocal music group exhibited increased activations in the frontal termination points of the left FAT during vocal music listening compared with the audiobook group from acute to 3 month poststroke stage. The increased activity correlated with the structural neuroplasticity changes in the left FAT. These results suggest that the beneficial effects of vocal music listening on poststroke language recovery are underpinned by structural neuroplasticity changes within the language network and extend our understanding of music-based interventions in stroke rehabilitation.
\end{abstract}

Key words: aphasia; DTI; fMRI; language; music; recovery

\section{Significance Statement}

Poststroke language deficits have a devastating effect on patients and their families. Current treatments yield highly variable outcomes, and the evidence for their long-term effects is limited. Patients often receive insufficient treatment that is predominantly given outside the optimal time window for brain plasticity. Poststroke vocal music listening improves language outcome, which is underpinned by neuroplasticity changes within the language network. Vocal music listening provides a complementary rehabilitation strategy that could be safely implemented in the early stages of stroke rehabilitation and seems to specifically target language symptoms and recovering language network.

Received April 13, 2021; accepted June 6, 2021; First published June 17, 2021.

The authors declare no competing financial interests.
Author contributions: A.J.S., P.R., V.L., J.S., R.P., A.R.-F., S.S., and T.S. designed research; A.J.S., V.L., J.S., R.P., and S.S. performed research; A.J.S., P.R., V.L., and T.S. analyzed data; A.J.S., P.R., V.L., J.S., R.P., A.R.-F., S.S., and T.S. wrote the paper. 


\section{Introduction}

Rapid aging of the population leads to a massive growth in the prevalence of stroke (Feigin et al., 2014), which incurs enormous socioeconomical challenges (Olesen et al., 2012). Poststroke aphasia-an impairment of speech production and/or comprehension-occurs in up to $40 \%$ of stroke patients (Pedersen et al., 2004) and has a devastating impact on the individual, decreasing quality of life more than any other stroke-induced impairment (Lam and Wodchis, 2010).

Language functions are underpinned by a left-lateralized network comprising frontal, temporal, and parietal brain regions and the white matter pathways interconnecting them (Hickok and Poeppel, 2007; Saur et al., 2008; Rauschecker and Scott, 2009; Alyahya et al., 2020; Hula et al., 2020). In poststroke language impairments, the language network is disrupted because of hypoperfusion and consequent brain tissue damage (Fox, 2018), and recovery relies on the ability of the spared neurons to remodel the injured network (Kiran et al., 2019). Aphasia treatments aim to achieve functional gains by promoting neuroplasticity processes within the language network (Cramer, 2008, 2018). Better aphasia outcomes have been associated with functional neuroplasticity changes within the language network, mainly in the left hemisphere, during both spontaneous recovery (Saur et al., 2006) and after treatments (Fridriksson, 2010; Fridriksson et al., 2012; Van Hees et al., 2014a; Hartwigsen and Saur, 2019). Studies evaluating treatment-related structural connectivity changes in aphasia are sparse, but have linked better outcomes with plasticity changes in the lefthemispheric white matter tracts (Van Hees et al., 2014b).

Current treatments have, however, shown highly variable outcomes and the evidence for their long-term effects is scarce (Brady et al., 2016). It is vital to pursue new rehabilitation methods that are inexpensive and both independent of and complementary to the traditional rehabilitation strategies. In this vein, music-based interventions have emerged as promising rehabilitation strategies in many neurologic diseases, including stroke (Winstein et al., 2016; Sihvonen et al., 2017c). In neurologic rehabilitation, music provides a multidomain stimulus that increases activity-dependent neuroplasticity in the brain and provides a fertile environment for recovery (Murphy and Corbett, 2009; Särkämö and Soto, 2012). In stroke patients, daily music listening during

This study was supported by the European Research Council (Grant 803466), the Academy of Finland (Grants 257077, 277693, 299044), Tyks Research Funding (Grant 13944), the Finnish Brain Research and Rehabilitation Foundation, the Finnish Brain Foundation, the Finnish Cultural Foundation (Grant 191230), the Orion Research Foundation, and the Signe and Ane Gyllenberg Foundation.

We thank the patients and their families for their participation, and the staff of the Tyks Neurocenter and rehabilitation hospitals in the Hospital District of Southwest Finland for collaboration.

Correspondence should be addressed to Aleksi J. Sihvonen at aleksi. sihvonen@helsinki.fi.

https://doi.org/10.1523/ENEURO.0158-21.2021

Copyright @ 2021 Sihvonen et al.

This is an open-access article distributed under the terms of the Creative Commons Attribution 4.0 International license, which permits unrestricted use, distribution and reproduction in any medium provided that the original work is properly attributed. the subacute poststroke stage has been found beneficial for improving cognitive and emotional recovery (Särkämö et al., 2008; Baylan et al., 2020) and increasing gray matter volume in frontolimbic regions (Särkämö et al., 2014) compared with standard care. Recently, using data pooled together from two randomized controlled trials (RCTs), we showed that the vocal (sung) component of music is crucial for its rehabilitative efficacy: compared with instrumental music and audiobooks, vocal music listening improved language recovery and verbal memory, especially in patients with aphasia, and was coupled with increased gray matter volume in temporal regions and enhanced functional connectivity of the default mode network (Sihvonen et al., 2020).

While vocal music listening promotes poststroke language recovery, little is known about the specific language-related neural mechanisms supporting this effect. An interesting hypothesis is that vocal music listening induces neuroplasticity effects on the language network, especially in the regions linked to connected speech after stroke (e.g., the left frontal regions and their underlying white matter tracts; Alyahya et al., 2020). Evaluating the possible neurobiological mediators of recovery and treatment effects is of great importance for improving our understanding of aphasia rehabilitation, and for optimizing current and future approaches (Copland, 2020).

The present study sought to unveil the neuroplasticity effects of vocal music in both the brain function and the structural connectivity of the language network. To do so, we assessed longitudinally a subsample of 38 stroke patients from our previous music intervention study (Sihvonen et al., 2020) using diffusion-weighted imaging (DWI) and task-related functional MRI (fMRI). We hypothesized that poststroke vocal music listening induces neuroplasticity changes in the language network that, in turn, underpin the enhanced recovery of language skills (Sihvonen et al., 2020).

\section{Materials and Methods}

\section{Subjects and study design}

Fifty stroke patients were recruited from 2013 to 2016 from the Turku University Hospital for a three-arm RCT (ClinicalTrials.gov: trial NCT01749709). Inclusion criteria were acute unilateral stroke; right handedness; age $<80$ years; capability to communicate in Finnish; residence in Southwest Finland; ability to cooperate; and normal hearing. Patients with prior neurologic or psychiatric disease or substance abuse were not included. The study was approved by the Ethics Committee of the Hospital District of Southwest Finland and performed in conformance with the Declaration of Helsinki. All patients gave informed consent, and received standard stroke treatment and rehabilitation. Baseline MRI scans and behavioral assessments were performed $<3$ weeks poststroke (mean, $12 \mathrm{~d}$; SD, 5.5). Patients were then randomly allocated to vocal music group (VMG; $N=17$ ), instrumental music group (IMG; $N=17$ ), and audiobook group (ABG; $N=16$ ). The randomization was stratified for lesion laterality (left/right) and performed as block randomization (10 blocks of three consecutive patients for 
Table 1: Baseline demographic and clinical characteristics of the patients

\begin{tabular}{|c|c|c|c|c|}
\hline & $\begin{array}{l}\text { Vocal music } \\
\text { group }(N=12)\end{array}$ & $\begin{array}{l}\text { Instrumental music } \\
\text { group }(N=15)\end{array}$ & $\begin{array}{l}\text { Audiobook } \\
\text { group }(N=11)\end{array}$ & $p$ Value \\
\hline Sex (male/female) & $5 / 7$ & $11 / 4$ & $7 / 4$ & $0.239\left(\chi^{2}\right)$ \\
\hline Education (years) & $14.7(3.6)$ & $13.8(3.9)$ & $12.5(4.4)$ & $0.450(F)$ \\
\hline \multicolumn{5}{|l|}{ Music background (prestroke) } \\
\hline Formal music training ${ }^{a}$ & $0.6(1.5)$ & $0.0(0.0)$ & $0.9(1.9)$ & $0.218(\mathrm{H})$ \\
\hline \multicolumn{5}{|l|}{ Clinical } \\
\hline Stroke type (infarct/haemorrhage) & $10 / 2$ & $9 / 6$ & $7 / 4$ & $0.398\left(\chi^{2}\right)$ \\
\hline Verbal fluency ${ }^{b}$ & $8.4(5.4)$ & $9.5(5.3)$ & $8.3(3.3)$ & $0.715(\mathrm{H})$ \\
\hline Naming $^{c}$ & $18.3(1.8)$ & $17.5(2.0)$ & $17.4(1.7)$ & $0.444(\mathrm{H})$ \\
\hline Auditory comprehension $^{d}$ & $27.5(5.5)$ & $27.7(3.5)$ & $24.6(5.1)$ & $0.112(\mathrm{H})$ \\
\hline Amusia overalle (no/yes) & $5 / 7$ & $9 / 6$ & $2 / 9$ & $0.103\left(\chi^{2}\right)$ \\
\hline
\end{tabular}

Data are the mean (SD), unless otherwise stated. Significant group differences are shown in bold. $F$, One-way ANOVA; $\mathrm{H}, \mathrm{Kruskal-Wallis} \mathrm{test;} \chi^{2}$, $\chi^{2}$ test.

a Likert scale 0-5 (0, never; 1 , rarely; 2, once a month; 3, once a week; 4, two to three times a week; 5 , daily).

b Classification based on Verbal Fluency Test.

${ }^{\mathrm{C}}$ Classification based on shortened Boston Naming test.

${ }^{\mathrm{d}}$ Classification based on shortened Token Test.

e Classification based on the MBEA Scale and Rhythm subtest average score ( $<75 \%$ cutoff).

${ }^{f}$ Classification based on the MBEA Scale subtest score ( $<73 \%$ cutoff).

${ }^{9}$ Classification based on the MBEA Rhythm subtest score ( $<77 \%$ cutoff).

left and right lesions), with the order within the blocks being drawn by a random number generator. The randomization list was generated by a laboratory engineer not involved in the data collection and the persons performing the patient recruitment had no access to it (allocation concealment). During follow-up, six patients were excluded because of refusal to participate at follow-up, and six patients because of incomplete MRI data. Thirtyeight of the remaining patients (15 female and 23 male; mean age, 56.1 years; SD, 13.4) completed the intervention and 3 month postintervention MRI and behavioral assessments, and were included in statistical analyses (VMG, $N=12$; IMG, $N=15 ; \mathrm{ABG}, \quad N=11$; Table 1). The groups did not differ between clinical variables (Table 1) such as stroke type $(p=0.398)$, lesion laterality $(p=0.676)$, or lesion volume $(p=0.712)$, nor did the study groups differ between the National Institutes of Health Stroke Scale scores at the acute stage $\left(F_{(22,38)}=0.872, p=0.627\right.$; Wilks' lambda $=$ 0.442 ; individual categories. $p=0.153-0.994)$. Patients with both ischemic and hemorrhagic strokes were included to reflect the real-world clinical population undergoing rehabilitation as well as to increase the generalization of the effects of this intervention. In clinical populations, the prevalence of poststroke cognitive impairments has not been shown to differ between ischemic and hemorrhagic strokes (Lo et al., 2019).

\section{Intervention}

After baseline assessments, each patient was contacted by a professional music therapist who informed them of their group allocation and interviewed them about prestroke leisure activities, including music listening and reading. Other researchers were blinded to the group allocation of the patients. The therapist provided the patients with a portable MP3 player, headphones, and a collection of listening material individually selected to match the music or literature preferences of the patient as closely as possible. The listening material was vocal music with sung lyrics in VMG, instrumental music (with no sung lyrics) in IMG, and narrated audiobooks (with no music) in ABG. All material was in a language that the patients understood best (mostly Finnish or English). The patients were trained in using the players, instructed to listen to the allocated material by themselves daily (minimum $1 \mathrm{~h} / \mathrm{d}$ ) for the following 2 months in the hospital or at home, and asked to keep a listening diary. During the 2 month intervention period, the music therapist kept regular contact with the patients to encourage listening, provide more material, and help with the equipment if needed.

\section{MRI data acquisition}

Patients were scanned on a $3 \mathrm{~T}$ Siemens Magnetom Verio scanner with a standard 12-channel head matrix coil at the Department of Radiology of Turku University Hospital. The MRI protocol comprised high-resolution T1-weighted anatomic images, DWI data $(T R=11,700$ ms; TE = 88 ms; acquisition matrix $=112 \times 112 ; 66$ axial slices; voxel size $=2.0 \times 2.0 \times 2.0 \mathrm{~mm}^{3}$ ) with one nondiffusion-weighted volume and 64 diffusion-weighted volumes $\left(b=1000 \mathrm{~s} / \mathrm{mm}^{2}\right)$, and task-fMRI using a single-shot T2*-weighted gradient-echo EPI sequence (280 functional volumes; 32 slices; slice thickness $=3.5$ $\mathrm{mm}$; TR $=2010 \mathrm{~ms}$; TE $=30 \mathrm{~ms}$; flip angle $=80^{\circ}$; voxel size $\left.=2.8 \times 2.8 \times 3.5 \mathrm{~mm}^{3}\right)$. 
During a block design task-fMRI, the patients were presented with $15 \mathrm{~s}$ excerpts of well known Finnish songs with (1) sung lyrics (vocal, 6 blocks) and (2) without sung lyrics (instrumental, 6 blocks), (3) well known Finnish poems (speech, 6 blocks), and (4) no auditory stimuli (rest, 18 blocks) through MR-compatible headphones using Presentation software (version 16.3, Neurobehavioral Systems). The order of the auditory blocks was randomized across subjects and time, and the rest blocks were presented in between the auditory blocks. Intervention listening material was not used in the task-fMRI excerpts.

\section{MRI data preprocessing}

MRI data were preprocessed using Statistical Parametric Mapping software [SPM8, Wellcome Department of Cognitive Neurology, UCL (www.fil.ion.ucl.ac.uk/spm/)] under MATLAB version 8.4.0. The $\mathrm{fMRI}$ images were initially realigned, and a mean image of the whole taskfMRI run was created. Individual images were reoriented according to the anterior commissure. Cost function masking was applied to achieve optimal normalization of the lesioned brain tissue, with no postregistration lesion shrinkage or out-of-brain distortion (Brett et al., 2001; Andersen et al., 2010; Ripollés et al., 2012). Cost function masking was performed by manually depicting the stroke lesions slice by slice to the individual T1 images using MRIcron software package (http://people.cas.sc.edu/rorden/mricron/index.html;

Rorden and Brett, 2000). All lesion tracing was conducted by one person (author A.J.S.) experienced in this matter (Sihvonen et al., 2016, 2017a). Task-fMRI data were normalized to Montreal Neurological Institute space using Unified Segmentation (Ashburner and Friston, 2005) and resampled into isotropic $2 \times 2 \times$ $2 \mathrm{~mm}^{3}$ voxel size. Finally, the preprocessed task-fMRI data were smoothed using an isotropic spatial filter $(\mathrm{FWHM}=8 \mathrm{~mm})$.

The statistical evaluation of the task-fMRI data was based on a least-squares estimation using the general linear model at both time points (acute/3 month). At the individual level, the different task conditions (vocal/instrumental/speech) were modeled with a box-car regressor waveform convolved with a canonical hemodynamic response function. Data were high-pass filtered to a maximum of $1 / 128 \mathrm{~Hz}$, and serial autocorrelations were estimated using an autoregressive model (AR[1] model). In addition, confounding factors from head movement were included in the model. A block-related design matrix was created including the conditions of interest (Vocal/ Instrumental/Speech). After model estimation, main effects for each condition against rest were calculated (e.g., Vocal > Rest).

\section{DTI data preprocessing}

The processing of DWI data started by correcting eddy current distortions and head motion using the FMRIB Software Library [FSL version 5.0.8, University of Oxford (www.fmrib.ox.ac.uk/fsl); Smith et al., 2004; Jenkinson et al., 2012]. Next, the gradient matrix was rotated using FSL fdt rotate bvecs to provide a more accurate estimate of diffusion tensor orientations (Leemans and Jones, 2009). Following this, brain extraction was performed using the Brain Extraction Tool (Smith, 2002). Then, the diffusion tensors were reconstructed using the linear least-squares algorithm included in Diffusion Toolkit version 0.6.2.2 (Ruopeng Wang, Van J. Wedeen (trackvis.org/dtk), Martinos Center for Biomedical Imaging, Massachusetts General Hospital, Charlestown, MA).

Dissections of individual white matter tracts were performed using TrackVis (version 0.6.0.1, Build 2015.04.07) following commonly used published guidelines for the number and positioning of the regions of interest (Catani et al., 2002; Sihvonen et al., 2017b). All analyses were performed by one person (author A.J.S.) experienced in virtual dissections (Sihvonen et al., 2017b). Deterministic tractography analysis focused on three white matter tracts integral to the language network and language skills (Catani et al., 2005, 2012; Dick and Tremblay, 2012; Dick et al., 2019; Alyahya et al., 2020): the left arcuate fasciculus (AF; long segment), the inferior fronto-occipital fasciculus (IFOF), and the left frontal aslant tract (FAT). After dissections, fractional anisotropy (FA) values of each tract, representing white matter integrity, were collected using MATLAB toolbox, "along-tract statistics" (Colby et al., 2012) and imported into IBM SPSS Statistics 27. Lower FA values in left-hemispheric tracts have been associated with greater aphasia severity (Rosso et al., 2015). Deterministic tractography dissections and placement of regions of interest have been described in detail previously (Sihvonen et al., 2017b).

\section{Language assessment}

Language assessment was performed in both time points (acute, 3 months) using the standard Verbal Fluency Test (Lezak et al., 2012); the shortened Token Test (De Renzi and Faglioni, 1978); and the shortened Boston Naming test (a 20 -item version including every third of the original 60 line drawings with a maximum score of 20; Morris et al., 1989; Laine et al., 1993), blinded to the group allocation of the patient. In our previous study (Sihvonen et al., 2020), the individual tests correlated significantly with each other (acute stage in all: $r=0.46-0.84, p<0.001$ ) and a summary score was calculated by adding up the raw test scores used in the analyses. To follow our previous study, and to maintain continuity and uniformity, as well as to conform the smaller sample size, the summary score was used in the current analyses.

\section{Statistical analyses}

In deterministic tractography analysis, multivariate ANOVA with change ( 3 month minus acute) in FA values of the left AF, IFOF, and FAT as dependent variables and Group as a factor was performed. Total brain volume (TBV) and cross-listening (i.e., listening to material not part of the protocol) were included as covariates in the analysis (Sihvonen et al., 2020). In addition, the groups 
A

Structural neuroplasticity

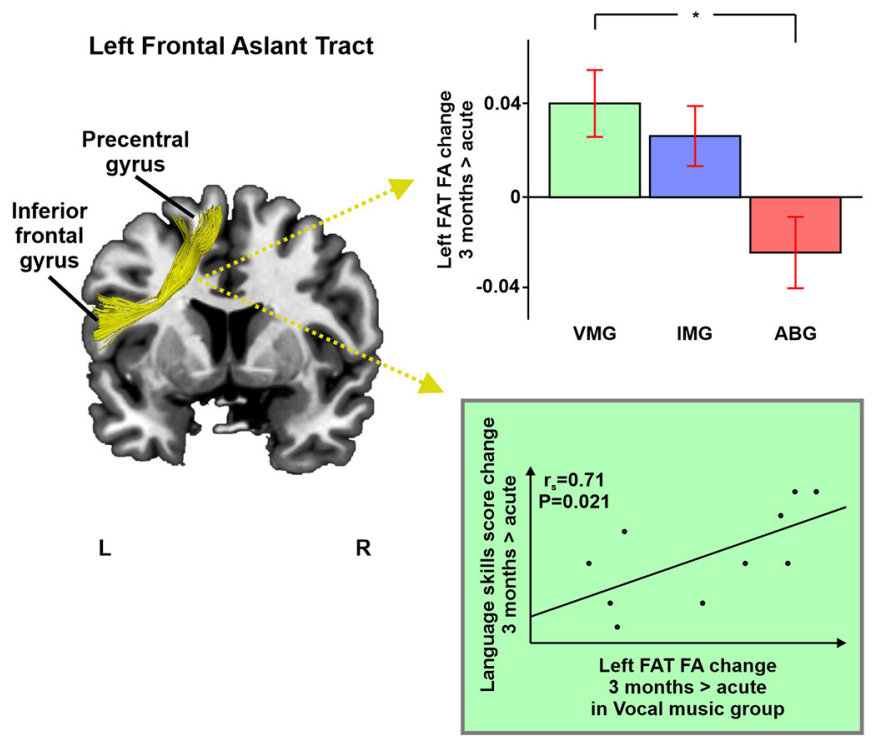

B

Functional neuroplasticity

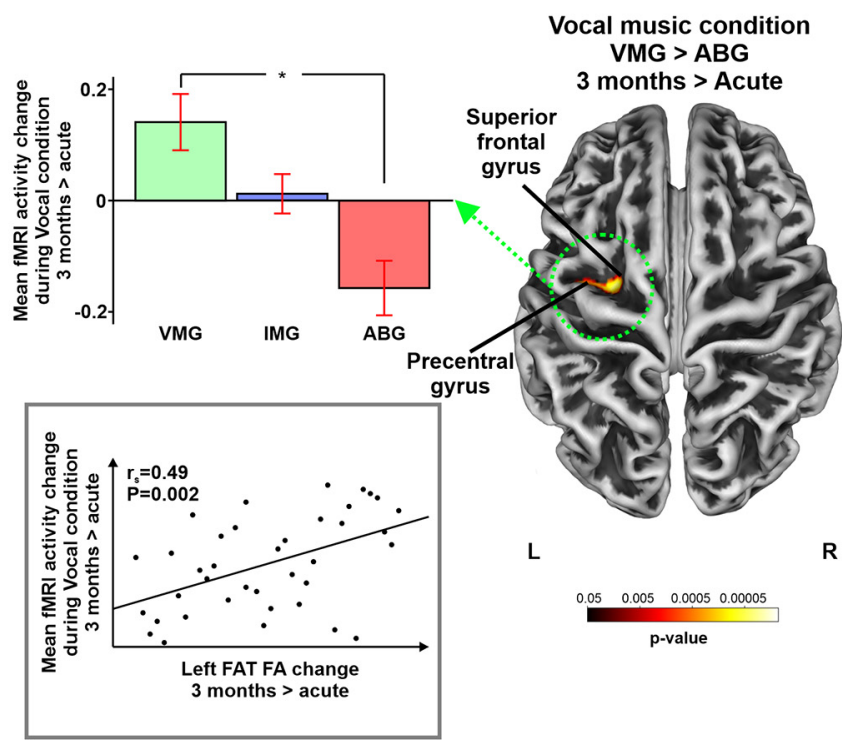

Figure 1. Structural and functional neuroplasticity changes (3 month $>$ Acute). $\boldsymbol{A}$, Significant tractography results showing increased FA in the left FAT for VMG $>$ ABG (3 month $>$ Acute). Correlations to change in language skills score within the Vocal music group are shown in the scatter plot. $\boldsymbol{B}$, Significant fMRI-task results showing increased activity between VMG and ABG (3 month $>$ Acute) during vocal music condition. Correlations to increased FA of the left FAT are shown in the scatter plot. Data reported in the histograms are the mean \pm SEM. * $p<0.017$. L, left; $R$, right.

showed significant differences in the prevalence of rhythm amusia $(p=0.028$; Table 1$)$, and therefore the Montreal Battery of Evaluation of Amusia (MBEA) Rhythm Subtest score (Peretz et al., 2003) was also included as a covariate in the analysis. Results were corrected for post hoc multiple comparisons using the Bonferroni adjustment.

Statistical analyses of the preprocessed task-fMRI data were conducted using SPM8. To evaluate longitudinal changes, three flexible factorial ANOVAs with Time (Acute/3 month) and Group (VMG/IMG/ABG) as factors were performed using the Vocal > Rest, Instrumental > Rest, and Speech $>$ Rest conditions. To focus analyses on the language network, analyses were constricted to the left hemisphere using an explicit mask. All task-fMRI result (spmT) maps were thresholded at an uncorrected $p<0.001$ at the voxel level, and standard SPM familywise error cluster-level correction based on random field theory with a $p$ value $<0.05$ was used (Eklund et al., 2016). Because of three conditions, $\alpha$-level was set to $p<0.017$, and only clusters surviving this threshold are reported. Similar to tractography, TBV, cross-listening, and MBEA Rhythm Subtest score were included as covariates in the task-fMRI analysis (Table 1).

Correlation analyses (Spearman, two-tailed) were performed between the significant tractography and fMRI changes ( 3 month $>$ Acute) and the changes in language skills.

\section{Data availability}

The data that support the findings of this study are available from the corresponding author, A.J.S., on reasonable request.

\section{Results}

\section{Structural neuroplasticity}

In the multivariate ANOVA, there was a statistically significant difference in the longitudinal change (3 month $>$ Acute) in the microstructural properties of the left-hemispheric tracts between the groups $\left(F_{(6,60)}=\right.$ 2.859; $p=0.016$; Wilks' lambda $=0.605$, partial $\eta^{2}=$ $0.222)$. Therefore, three separate univariate ANOVAs (i.e., one for each tract) were performed, and $\alpha$-level was set to $p<0.017$.

Separate univariate ANOVAs for each tract revealed a significant effect on the FA values of the left FAT, indicating that the longitudinal change (3 month $>$ Acute) in the microstructural properties of this tract differed between the groups $\left(F_{(2,32)}=4.819, p=0.015\right.$; partial $\left.\eta^{2}=0.231\right)$. Post hoc $t$ tests corrected for multiple comparisons (Bonferroni) revealed that the VMG showed a significantly greater increase in FA values over time than the ABG $(p=0.017$; Fig. $1 A)$, whereas there were no significant differences between the IMG and ABG or VMG and IMG. Importantly, the FA change in the left FAT correlated with improved language skills $\left(r_{\mathrm{s}}=0.71, p=0.021\right)$ within the VMG. There were no significant interactions for the other tracts (AF, IFOF).

\section{Functional neuroplasticity}

Similarly, there was a significant Group $\times$ Time interaction for the Vocal > Rest condition revealing that the VMG showed greater longitudinal (3 month $>$ Acute) activation increase than the $A B G$ in a specific left frontal cluster $(p=0.016 ; T=4.63 ;$ size $=216$ voxels $)$ located in the 
superior/middle frontal gyrus and the precentral gyrus (Fig. 1B). The increased activity in the significant cluster correlated with the increased FA in the left FAT $r_{\mathrm{s}}=0.49, \quad p=0.002$ ) across the whole sample. Correlation between the increased activity and improved language skills was nonsignificant. No other significant interactions were detected for the other task conditions (Instrumental > Rest, Speech > Rest).

\section{Discussion}

This study set out to determine the poststroke vocal music listening induced functional and structural neuroplasticity changes in the language network possibly supporting the improved language skills. Our two main findings were that, compared with listening to audiobooks (1) daily poststroke vocal music listening enhanced left FAT structural connectivity, which was linked to better recovery of language skills; and (2) vocal music listening led to increased stimulus-specific functional changes in the superior frontal termination areas of the left FAT that were linked to improved structural connectivity in the left FAT. The present study not only extends previous results on the rehabilitative effects of music listening after stroke (Särkämö et al., 2008, 2014; Baylan et al., 2020; Sihvonen et al., 2020), but also reveals novel information about the neural mechanisms (i.e., functional and structural reorganization of key regions within the language network) that support language recovery in stroke via vocal music listening. This evidence is important in evaluating treatment mediators of music-based rehabilitation strategies (Sihvonen et al., 2017c), and in improving our understanding of aphasia rehabilitation.

Connecting the inferior frontal gyrus with dorsomedial frontal areas and anterior cingulate cortex, the left FAT has recently been recognized as an important tract for speech production (Catani et al., 2012; Thiebaut de Schotten et al., 2012; Dick et al., 2014, 2019; Sierpowska et al., 2015). Damage to the FAT underlies disease-related speech impairments in patients with poststroke aphasia (Basilakos et al., 2014; Halai et al., 2017; Alyahya et al., 2020) and primary progressive aphasia (Catani et al., 2013; Mandelli et al., 2014), as well as in patients with resected frontal gliomas (Kinoshita et al., 2015). Moreover, neuroplasticity changes in the inferior frontal and dorsomedial termination points of the left FAT have been shown to underpin better aphasia outcomes both after targeted rehabilitation and also in patients showing spontaneous recovery (Saur et al., 2006; Schevenels et al., 2020). En masse, while studies evaluating direct aphasia treatmentrelated structural changes in the left FAT are lacking, its role in regaining language functions after poststroke aphasia as well as a potential target for aphasia treatments stands to reason. The present study suggests that this avenue for poststroke language recovery could be targeted by listening to vocal music.

The sensory and motor environment during the acute stroke stage has a crucial role in the recovery. However, in clinical practice, patients often receive rehabilitation in suboptimal intensity, frequency, and timing (Murphy and Corbett, 2009; Foley et al., 2012), and remain largely inactive and unstimulated during the critical acute stage (Bernhardt et al., 2004; De Wit et al., 2005). In other words, the prerequisites for poststroke rehabilitation exploiting activity-dependent neural plasticity are often not met (Cramer et al., 2011). Music listening could respond to these unmet needs of recovering stroke patients. First, music listening serves as a multimodal stimulus, akin to "enriched environment" where neural stimulation is achieved by increasing stimuli from the physical and social surroundings during the rehabilitation (Nithianantharajah and Hannan, 2006). Studies on healthy subjects have revealed that mere music listening induces a widespread activation pattern in the brain (Schmithorst, 2005; Samson et al., 2011; Alluri et al., 2012; Zatorre and Salimpoor, 2013; Koelsch, 2014). In acute stroke patients, music listening activates a similar network of brain regions (Sihvonen et al., 2017d). This increased neural stimulation supports neural plasticity in the recovering brain by increasing, for example, dendritic spine density and neurotrophic factor levels (Nithianantharajah and Hannan, 2006).

Second, language and music processing have been shown to be supported by common neural networks (Maess et al., 2001; Koelsch et al., 2002; Callan et al., 2006; Schön et al., 2010; Kunert et al., 2015); that is, language network engagement can be modulated by music. This modulatory effect can be enhanced by listening to music with sung lyrics (i.e., vocals), which binds linguistic and musical information into a unified representation: vocal music engages bilateral frontotemporal areas more extensively than speech (Callan et al., 2006; Schön et al., 2010) or music without vocals (i.e., instrumental music; Brattico et al., 2011; Alluri et al., 2013), even in patients with acute stroke (Sihvonen et al., 2017d). Importantly, vocal music engages the left inferior and dorsomedial frontal termination areas of the left FAT, which have been implicated in the auditory-motor processing of music (Zatorre et al., 2007) and singing (Callan et al., 2006). Crucially, the left dorsomedial frontal areas (superior frontal gyrus and anterior cingulate) showed increased gray matter volume after the music listening intervention in our previous stroke study (Särkämö et al., 2014), and the anterior cingulate is also the frontal hub of the default mode network where we previously reported enhanced functional connectivity induced by the vocal music listening (Sihvonen et al., 2020). The present results elaborate the activity-dependent neuroplasticity effects of poststroke vocal music listening by elucidating its effects on modulating communal neural structures for speech and music, underpinning language recovery after stroke.

Regarding the real-world shortcomings in rehabilitation intensity, frequency, and timing, poststroke vocal music listening could be implemented with minimal professional input early in the rehabilitation process (i.e., acute stage). One hour daily patient-led music listening has been shown to be enough in terms of intensity to produce behavioral (Särkämö et al., 2008; Sihvonen et al., 2020) and neuroplasticity (Särkämö et al., 2014; Sihvonen et al., 2020) gains, and, if implemented, could increase the received daily rehabilitation (De Wit et al., 2005) and possibly provide better aphasia outcomes (Bhogal et al., 2003). 
In turn, this could provide better long-term outcomes by increasing mood and ameliorating social isolation (Doogan et al., 2018).

The present study has some potential limitations. While the randomization to groups was stratified for lesion laterality, the groups showed significant differences for the prevalence of rhythm amusia, the inability to perceive musical rhythm. However, the number of patients with rhythm amusia in each group was largely similar (ranging from 8 to 11). Moreover, the prevalence of amusia and aphasia in the current sample was similar to those in previous studies (Sihvonen et al., 2019), making it representative of the real-world population. Most importantly, despite these group differences, vocal music listening was still effective. To fill the inclusion criteria, patients were required that have some degree of verbal communication and speech comprehension. This means that patients with global or severe aphasia were not able to participate, and aphasic patients in the current study have mild to moderate aphasia. This impedes us from making any conclusion on the effects of vocal music listening in severe forms of poststroke language impairments, and in the future, these effects should be studied. This very limitation can also contribute to the observed pattern of results: in different forms and severities of aphasia, improved outcomes can be underpinned by structural neuroplasticity changes in white matter pathways other than the left FAT (Alyahya et al., 2020; Hula et al., 2020; Gajardo-Vidal et al., 2021). How vocal music listening can target those and possibly mediate recovery is as yet unknown. Moreover, the current sample size prevented us from performing separate analyses for aphasic and nonaphasic patients.

Future research on the effects and the most active ingredients of music listening in poststroke rehabilitation is still needed. One crucial aspect of music is its capacity to evoke and regulate emotions, provide joy and comfort, and relieve stress (Saarikallio, 2011). Emotionally engaging music activates multiple brain circuits related to dopaminergic signaling reward and emotion, the engagement of which has been shown to be directly proportional to the intensity of the experience (Blood and Zatorre, 2001; Salimpoor et al., 2011; Ferreri et al., 2019). The engagement of crucial brain regions related to dopamine, motivation, and pleasure could partly explain the cognitiveemotional gains induced by music listening in neurologic rehabilitation (Sihvonen et al., 2017c). Importantly, the music in the study was self-selected by the patients to maximize personal relevance and emotional arousal, and this could have further enhanced the benefits of poststroke music listening. Future studies should include online (i.e., while the patients listen to music as part of their daily therapy) subjective (e.g., self-reports of pleasure) and objective (e.g., physiological responses via wearable technology that does not hinder the musical experience; Pelofi et al., 2021) measures evaluating the emotional arousal and pleasure of the music material. Furthermore, vocal music listening could be used as an adjuvant therapy in connection with traditional speech therapy to provide neural stimulation and fertile ground for recovery (Nithianantharajah and Hannan, 2006). As a receptive form of music-based rehabilitation, music listening could also support the effects of melodic intonation therapy (Albert et al., 1973), an active, singing-based treatment, the goal of which is to restore propositional speech, that has been related to neuroplasticity effects in the right AF (Schlaug et al., 2009). Furthermore, it would be extremely interesting to investigate whether the beneficial effects of vocal music listening are accessible in patients with different lesion locations affecting the language system (i.e., temporoparietal and frontal strokes; Stockert et al., 2020) and in patients with different aphasia severities and subtypes. Future studies using larger samples should assess the effect that music listening has on more specific language- and auditory-related functions and how this interacts with hemispheric damage. This is of particular importance for language-related functions thought to be supported by bilateral regions in the temporal cortex (Hickok and Poeppel, 2007).

In conclusion, the present results suggest that the positive effects of music listening on stroke recovery are underpinned by structural and functional reorganization of the left hemisphere language network for vocal music. Clinically, the results provide further evidence that vocal music listening is a feasible tool to stimulate the language network and promote language recovery after stroke.

\section{References}

Albert ML, Sparks RW, Helm NA (1973) Melodic intonation therapy for aphasia. Arch Neurol 29:130-131.

Alluri V, Toiviainen P, Jääskeläinen IP, Glerean E, Sams M, Brattico E (2012) Large-scale brain networks emerge from dynamic processing of musical timbre, key and rhythm. Neuroimage 59:3677-3689.

Alluri V, Toiviainen P, Lund TE, Wallentin M, Vuust P, Nandi AK, Ristaniemi T, Brattico E (2013) From vivaldi to beatles and back: predicting lateralized brain responses to music. Neuroimage 83:627-636.

Alyahya RSW, Halai AD, Conroy P, Lambon MA (2020) A unified model of post-stroke language deficits including discourse production and their neural correlates. Brain 143:1541-1554.

Andersen SM, Rapcsak SZ, Beeson PM (2010) Cost function masking during normalization of brains with focal lesions: still a necessity? Neuroimage 53:78-84.

Ashburner J, Friston KJ (2005) Unified segmentation. Neuroimage 26:839-851.

Basilakos A, Fillmore PT, Rorden C, Guo D, Bonilha L, Fridriksson J (2014) Regional white matter damage predicts speech fluency in chronic post-stroke aphasia. Front Hum Neurosci 8:845.

Baylan S, Haig C, MacDonald M, Stiles C, Easto J, Thomson M, Cullen B, Quinn TJ, Stott D, Mercer SW, Broomfield NM, Murray H, Evans JJ (2020) Measuring the effects of listening for leisure on outcome after stroke (MELLO): a pilot randomized controlled trial of mindful music listening. Int J Stroke 15:149-158.

Bernhardt J, Dewey H, Thrift A, Donnan G (2004) Inactive and alone: physical activity within the first 14 days of acute stroke unit care. Stroke 35:1005-1009.

Bhogal SK, Teasell R, Speechley M (2003) Intensity of aphasia therapy, impact on recovery. Stroke 34:987-992.

Blood AJ, Zatorre RJ (2001) Intensely pleasurable responses to music correlate with activity in brain regions implicated in reward and emotion. Proc Natl Acad Sci U S A 98:11818-11823.

Brady MC, Kelly H, Godwin J, Enderby P, Campbell P (2016) Speech and language therapy for aphasia following stroke. Cochrane Database Syst Rev 2016:CD000425. 
Brattico E, Alluri V, Bogert B, Jacobsen T, Vartiainen N, Nieminen S, Tervaniemi M (2011) A functional MRI study of happy and sad emotions in music with and without lyrics. Front Psychol 2:308.

Brett M, Leff AP, Rorden C, Ashburner J (2001) Spatial normalization of brain images with focal lesions using cost function masking. Neuroimage 14:486-500.

Callan DE, Tsytsarev V, Hanakawa T, Callan AM, Katsuhara M, Fukuyama H, Turner R (2006) Song and speech: brain regions involved with perception and covert production. Neuroimage 31:1327-1342.

Catani M, Howard RJ, Pajevic S, Jones DK (2002) Virtual in vivo interactive dissection of white matter fasciculi in the human brain. Neuroimage 17:77-94.

Catani M, Jones DK, Ffytche DH (2005) Perisylvian language networks of the human brain. Ann Neurol 57:8-16.

Catani M, Dell'acqua F, Vergani F, Malik F, Hodge H, Roy P, Valabregue R, Thiebaut de Schotten M (2012) Short frontal lobe connections of the human brain. Cortex 48:273-291.

Catani M, Mesulam MM, Jakobsen E, Malik F, Martersteck A, Wieneke C, Thompson CK, Thiebaut de Schotten M, Dell'Acqua F, Weintraub S, Rogalski E (2013) A novel frontal pathway underlies verbal fluency in primary progressive aphasia. Brain 136:26192628.

Colby JB, Soderberg L, Lebel C, Dinov ID, Thompson PM, Sowell ER (2012) Along-tract statistics allow for enhanced tractography analysis. Neuroimage 59:3227-3242.

Copland DA (2020) Elizabeth Usher Memorial Lecture: lost in translation? Challenges and future prospects for a neurobiological approach to aphasia rehabilitation. Int J Speech Lang Pathol 22:270280.

Cramer SC (2008) Repairing the human brain after stroke. II. Restorative therapies. Ann Neurol 63:549-560.

Cramer SC (2018) Treatments to promote neural repair after stroke. J Stroke 20:57-70.

Cramer SC, Sur M, Dobkin BH, O'Brien C, Sanger TD, Trojanowski JQ, Rumsey JM, Hicks R, Cameron J, Chen D, Chen WG, Cohen LG, deCharms C, Duffy CJ, Eden GF, Fetz EE, Filart R, Freund M, Grant SJ, Haber S, et al. (2011) Harnessing neuroplasticity for clinical applications. Brain 134:1591-1609.

De Renzi E, Faglioni P (1978) Normative data and screening power of a shortened version of the token test. Cortex 14:41-49.

De Wit L, Putman K, Dejaeger E, Baert I, Berman P, Bogaerts K, Brinkmann N, Connell L, Feys H, Jenni W, Kaske C, Lesaffre E, Leys M, Lincoln N, Louckx F, Schuback B, Schupp W, Smith B, De Weerdt W (2005) Use of time by stroke patients: a comparison of four European rehabilitation centers. Stroke 36:1977-1983.

Dick AS, Tremblay P (2012) Beyond the arcuate fasciculus: consensus and controversy in the connectional anatomy of language. Brain 135:3529-3550.

Dick AS, Bernal B, Tremblay P (2014) The language connectome: new pathways, new concepts. Neuroscientist 20:453-467.

Dick AS, Garic D, Graziano P, Tremblay P (2019) The frontal aslant tract (FAT) and its role in speech, language and executive function. Cortex 111:148-163.

Doogan C, Dignam J, Copland D, Leff A (2018) Aphasia recovery: when, how and who to treat? Curr Neurol Neurosci Rep 18:90.

Eklund A, Nichols TE, Knutsson H (2016) Cluster failure: why fMRI inferences for spatial extent have inflated false-positive rates. Proc Natl Acad Sci U S A 113:7900-7905.

Feigin VL, Forouzanfar MH, Krishnamurthi R, Mensah GA, Connor M, Bennett DA, Moran AE, Sacco RL, Anderson L, Truelsen T, O'Donnell M, Venketasubramanian N, Barker-Collo S, Lawes CMM, Wang W, Shinohara Y, Witt E, Ezzati M, Naghavi M, Murray C (2014) Global and regional burden of stroke during 1990-2010: findings from the Global Burden of Disease Study 2010. Lancet 383:245-255.

Ferreri L, Mas-Herrero E, Zatorre RJ, Ripollés P, Gomez-Andres A, Alicart H, Olivé G, Marco-Pallarés J, Antonijoan RM, Valle M, Riba $\mathrm{J}$, Rodriguez-Fornells A (2019) Dopamine modulates the reward experiences elicited by music. Proc Natl Acad Sci U S A 116:3793-3798.

Foley N, McClure JA, Meyer M, Salter K, Bureau Y, Teasell R (2012) Inpatient rehabilitation following stroke: amount of therapy received and associations with functional recovery. Disabil Rehabil 34:2132-2138.

Fox MD (2018) Mapping symptoms to brain networks with the human connectome. N Engl J Med 379:2237-2245.

Fridriksson $\mathrm{J}$ (2010) Preservation and modulation of specific left hemisphere regions is vital for treated recovery from anomia in stroke. J Neurosci 30:11558-11564.

Fridriksson J, Richardson JD, Fillmore P, Cai B (2012) Left hemisphere plasticity and aphasia recovery. Neuroimage 60:854-863.

Gajardo-Vidal A, Lorca-Puls DL, Team P, Warner H, Pshdary B, Crinion JT, Leff AP, Hope TMH, Geva S, Seghier ML, Green DW, Bowman H, Price CJ (2021) Damage to Broca's area does not contribute to long-term speech production outcome after stroke. Brain 144:817-832.

Halai AD, Woollams AM, Lambon Ralph MA (2017) Using principal component analysis to capture individual differences within a unified neuropsychological model of chronic post-stroke aphasia: revealing the unique neural correlates of speech fluency, phonology and semantics. Cortex 86:275-289.

Hartwigsen G, Saur D (2019) Neuroimaging of stroke recovery from aphasia-insights into plasticity of the human language network. Neuroimage 190:14-31.

Hickok G, Poeppel D (2007) The cortical organization of speech processing. Nat Rev Neurosci 8:393-402.

Hula WD, Panesar S, Gravier ML, Yeh FC, Dresang HC, Dickey MW, Fernandez-Miranda JC (2020) Structural white matter connectometry of word production in aphasia: an observational study. Brain 143:2532-2544.

Jenkinson M, Beckmann CF, Behrens TEJ, Woolrich MW, Smith SM (2012) FSL. Neuroimage 62:782-790.

Kinoshita M, de Champfleur NM, Deverdun J, Moritz-Gasser S, Herbet G, Duffau H (2015) Role of fronto-striatal tract and frontal aslant tract in movement and speech: an axonal mapping study. Brain Struct Funct 220:3399-3412.

Kiran S, Meier EL, Johnson JP (2019) Neuroplasticity in aphasia: a proposed framework of language recovery. J Speech Lang Hear Res 62:3973-3985.

Koelsch S (2014) Brain correlates of music-evoked emotions. Nat Rev Neurosci 15:170-180.

Koelsch S, Gunter TC, Cramon DY, Zysset S, Lohmann G, Friederici AD (2002) Bach speaks: a cortical "language-network" serves the processing of music. Neuroimage 17:956-966.

Kunert R, Willems RM, Casasanto D, Patel AD, Hagoort P (2015) Music and language syntax interact in Broca's area: an fMRI study. PLoS One 10:e0141069.

Laine M, Goodglass H, Niemi J, Koivuselka-Sallinen P, Tuomainen J, Marttila R (1993) Adaptation of the Boston diagnostic aphasia examination and the boston naming test into finnish. Logoped Phoniatr Vocol 18:83-92.

Lam JMC, Wodchis WP (2010) The relationship of 60 disease diagnoses and 15 conditions to preference-based health-related quality of life in Ontario hospital-based long-term care residents. Med Care 48:380-387.

Leemans A, Jones DK (2009) The B-matrix must be rotated when correcting for subject motion in DTI data. Magn Reson Med 61:1336-1349.

Lezak MD, Howieson DB, Bigler ED, Tranel D (2012) Neuropsychological assessment, Ed 5. New York: Oxford UP.

Lo JW, Crawford JD, Desmond DW, Godefroy O, Jokinen H, Mahinrad S, Bae H-J, Lim J-S, Köhler S, Douven E, Staals J, Chen C, Xu X, Chong EJ, Akinyemi RO, Kalaria RN, Ogunniyi A, Barbay M, Roussel M, Lee B-C, et al (2019) Profile of and risk factors for poststroke cognitive impairment in diverse ethnoregional groups. Neurology 93:E2257-E2271. 
Maess B, Koelsch S, Gunter TC, Friederici AD (2001) Musical syntax is processed in Broca's area: an MEG study. Nat Neurosci 4:540545.

Mandelli ML, Caverzasi E, Binney RJ, Henry ML, Lobach I, Block N, Amirbekian B, Dronkers N, Miller BL, Henry RG, Gorno-Tempini ML (2014) Frontal white matter tracts sustaining speech production in primary progressive aphasia. J Neurosci 34:9754-9767.

Morris JC, Heyman A, Mohs RC, Hughes JP, van Belle G, Fillenbaum G, Mellits ED, Clark C (1989) The Consortium to Establish a Registry for Alzheimer's Disease (CERAD). Part I. Clinical and neuropsychological assessment of Alzheimer's disease. Neurology 39:1159-1165.

Murphy TH, Corbett D (2009) Plasticity during stroke recovery: from synapse to behaviour. Nat Rev Neurosci 10:861-872.

Nithianantharajah J, Hannan AJ (2006) Enriched environments, experience-dependent plasticity and disorders of the nervous system. Nat Rev Neurosci 7:697-709.

Olesen J, Gustavsson A, Svensson M, Wittchen HU, Jönsson B (2012) The economic cost of brain disorders in Europe. Eur J Neurol 19:155-162.

Pedersen PM, Vinter K, Olsen TS (2004) Aphasia after stroke: type, severity and prognosis: the Copenhagen aphasia study. Cerebrovasc Dis 17:35-43.

Pelofi C, Goldstein M, Bevilacqua D, McPhee M, Abrams E, Ripollés P (2021) CHILLER: a computer human interface for the live labelling of emotional responses. Paper presented at 2021 New Interfaces for Musical Expression, Shanghai, People's Republic of China, June.

Peretz I, Champod AS, Hyde K (2003) Varieties of musical disorders: the montreal battery of evaluation of amusia. Ann N Y Acad Sci 999:58-75.

Rauschecker JP, Scott SK (2009) Maps and streams in the auditory cortex: nonhuman primates illuminate human speech processing. Nat Neurosci 12:718-724.

Ripollés P, Marco-Pallarés J, de Diego-Balaguer R, Miró J, Falip M, Juncadella M, Rubio F, Rodriguez-Fornells A (2012) Analysis of automated methods for spatial normalization of lesioned brains. Neuroimage 60:1296-1306.

Rorden C, Brett M (2000) Stereotaxic display of brain lesions. Behav Neurol 12:191-200.

Rosso C, Vargas P, Valabregue R, Arbizu C, Henry-Amar F, Leger A, Lehéricy S, Samson Y (2015) Aphasia severity in chronic stroke patients: a combined disconnection in the dorsal and ventral language pathways. Neurorehabil Neural Repair 29:287-295.

Saarikallio S (2011) Music as emotional self-regulation throughout adulthood. Psychol Music 39:307-327.

Salimpoor VN, Benovoy M, Larcher K, Dagher A, Zatorre RJ (2011) Anatomically distinct dopamine release during anticipation and experience of peak emotion to music. Nat Neurosci 14:257-264.

Samson F, Zeffiro TA, Toussaint A, Belin P (2011) Stimulus complexity and categorical effects in human auditory cortex: an activation likelihood estimation meta-analysis. Front Psychol 1:241.

Särkämö T, Soto D (2012) Music listening after stroke: beneficial effects and potential neural mechanisms. Ann N Y Acad Sci 1252:266-281.

Särkämö T, Tervaniemi M, Laitinen S, Forsblom A, Soinila S, Mikkonen M, Autti T, Silvennoinen HM, Erkkilä J, Laine M, Peretz I, Hietanen M (2008) Music listening enhances cognitive recovery and mood after middle cerebral artery stroke. Brain 131:866-876.

Särkämö T, Ripollés $P$, Vepsäläinen $H$, Autti T, Silvennoinen HM, Salli E, Laitinen S, Forsblom A, Soinila S, Rodríguez-Fornells A (2014) Structural changes induced by daily music listening in the recovering brain after middle cerebral artery stroke: a voxel-based morphometry study. Front Hum Neurosci 8:245.

Saur D, Lange R, Baumgaertner A, Schraknepper V, Willmes K, Rijntjes M, Weiller C (2006) Dynamics of language reorganization after stroke. Brain 129:1371-1384.

Saur D, Kreher BW, Schnell S, Kümmerer D, Kellmeyer P, Vry M-S, Umarova R, Musso M, Glauche V, Abel S, Huber W, Rijntjes M,
Hennig J, Weiller C (2008) Ventral and dorsal pathways for language. Proc Natl Acad Sci U S A 105:18035-18040.

Schevenels K, Price CJ, Zink I, De Smedt B, Vandermosten M (2020) $A$ review on treatment-related brain changes in aphasia. Neurobiol Lang 1:402-433.

Schlaug G, Marchina S, Norton A (2009) Evidence for plasticity in white-matter tracts of patients with chronic Broca's aphasia undergoing intense intonation-based speech therapy. Ann N Y Acad Sci 1169:385-394.

Schmithorst VJ (2005) Separate cortical networks involved in music perception: preliminary functional MRI evidence for modularity of music processing. Neuroimage 25:444-451.

Schön D, Gordon R, Campagne A, Magne C, Astésano C, Anton JL, Besson M (2010) Similar cerebral networks in language, music and song perception. Neuroimage 51:450-461.

Sierpowska J, Gabarrós A, Fernandez-Coello A, Camins A, Castañer S, Juncadella M, de Diego-Balaguer R, RodríguezFornells A (2015) Morphological derivation overflow as a result of disruption of the left frontal aslant white matter tract. Brain Lang 142:54-64.

Sihvonen AJ, Leo V, Ripollés P, Lehtovaara T, Ylönen A, Rajanaro $P$, Laitinen S, Forsblom A, Saunavaara J, Autti T, Laine M, Rodríguez-Fornells A, Tervaniemi M, Soinila S, Särkämö T (2020) Vocal music enhances memory and language recovery after stroke: pooled results from two RCTs. Ann Clin Transl Neurol 7:2272-2287.

Sihvonen AJ, Ripollés P, Leo V, Rodríguez-Fornells A, Soinila S, Särkämö T (2016) Neural basis of acquired amusia and its recovery after stroke. J Neurosci 36:8872-8881.

Sihvonen AJ, Ripollés P, Rodríguez-Fornells A, Soinila S, Särkämö T (2017a) Revisiting the neural basis of acquired amusia: lesion patterns and structural changes underlying amusia recovery. Front Neurosci 11:426.

Sihvonen AJ, Ripollés $P$, Särkämö T, Leo V, Rodríguez-Fornells A, Saunavaara J, Parkkola R, Soinila S (2017b) Tracting the neural basis of music: deficient structural connectivity underlying acquired amusia. Cortex 97:255-273.

Sihvonen AJ, Särkämö T, Leo V, Tervaniemi M, Altenmüller E, Soinila $S(2017$ c) Music-based interventions in neurological rehabilitation. Lancet Neurol 16:648-660.

Sihvonen AJ, Särkämö T, Ripollés P, Leo V, Saunavaara J, Parkkola R, Rodríguez-Fornells A, Soinila S (2017d) Functional neural changes associated with acquired amusia across different stages of recovery after stroke. Sci Rep 7:11390.

Sihvonen AJ, Särkämö T, Rodríguez-Fornells A, Ripollés $P$, Münte TF, Soinila S (2019) Neural architectures of music-insights from acquired amusia. Neurosci Biobehav Rev 107:104-114.

Smith SM (2002) Fast robust automated brain extraction. Hum Brain Mapp 17:143-155.

Smith SM, Jenkinson M, Woolrich MW, Beckmann CF, Behrens TEJ, Johansen-Berg H, Bannister PR, De Luca M, Drobnjak I, Flitney DE, Niazy RK, Saunders J, Vickers J, Zhang Y, De Stefano N, Brady JM, Matthews PM (2004) Advances in functional and structural MR image analysis and implementation as FSL. Neuroimage 23:S208-S219.

Stockert A, Wawrzyniak M, Klingbeil J, Wrede K, Kümmerer D, Hartwigsen G, Kaller CP, Weiller C, Saur D (2020) Dynamics of language reorganization after left temporo-parietal and frontal stroke. Brain 143:844-861.

Thiebaut de Schotten M, Dell'Acqua F, Valabregue R, Catani M (2012) Monkey to human comparative anatomy of the frontal lobe association tracts. Cortex 48:82-96.

Van Hees S, McMahon K, Angwin A, de Zubicaray G, Copland DA (2014a) Neural activity associated with semantic versus phonological anomia treatments in aphasia. Brain Lang 129:47-57.

Van Hees S, McMahon K, Angwin A, De Zubicaray G, Read S, Copland DA (2014b) Changes in white matter connectivity following therapy for anomia post stroke. Neurorehabil Neural Repair 28:325-334. 
Winstein CJ, Stein J, Arena R, Bates B, Cherney LR, Cramer SC, Deruyter F, Eng JJ, Fisher B, Harvey RL, Lang CE, MacKay-Lyons M, Ottenbacher KJ, Pugh S, Reeves MJ, Richards LG, Stiers W, Zorowitz RD (2016) Guidelines for adult stroke rehabilitation and recovery: a guideline for healthcare professionals from the American Heart Association/American Stroke Association. Stroke 47:e98-e169.
Zatorre RJ, Salimpoor VN (2013) From perception to pleasure: music and its neural substrates. Proc Natl Acad Sci U S A 110:1043010437.

Zatorre RJ, Chen JL, Penhune VB (2007) When the brain plays music: auditory-motor interactions in music perception and production. Nat Rev Neurosci 8:547-558. 